\title{
WASP-39b: a highly inflated Saturn-mass planet orbiting a late G-type star ${ }^{\star}$
}

\author{
F. Faedi ${ }^{1}$, S. C. C. Barros ${ }^{1}$, D. R. Anderson ${ }^{2}$, D. J. A. Brown ${ }^{3}$, A. Collier Cameron ${ }^{3}$, D. Pollacco ${ }^{1}$, I. Boisse $^{4,5}$, \\ G. Hébrard ${ }^{4,6}$, M. Lendl ${ }^{7}$, T. A. Lister ${ }^{8}$, B. Smalley ${ }^{2}$, R. A. Street ${ }^{8}$, A. H. M. J. Triaud ${ }^{7}$, J. Bento ${ }^{9}$, F. Bouchy ${ }^{4,6}$, \\ O. W. Butters ${ }^{10}$, B. Enoch $^{3}$, C. A. Haswell ${ }^{11}$, C. Hellier ${ }^{2}$, F. P. Keenan ${ }^{1}$, G. R. M. Miller ${ }^{3}$, V. Moulds ${ }^{1}$, C. Moutou $^{12}$, \\ A. J. Norton ${ }^{11}$, D. Queloz ${ }^{7}$, A. Santerne ${ }^{4,6}$, E. K. Simpson ${ }^{1}$, I. Skillen ${ }^{13}$, A. M. S. Smith ${ }^{2}$, S. Udry ${ }^{7}$, C. A. Watson ${ }^{1}$, \\ R. G. West ${ }^{10}$, and P. J. Wheatley ${ }^{9}$
}

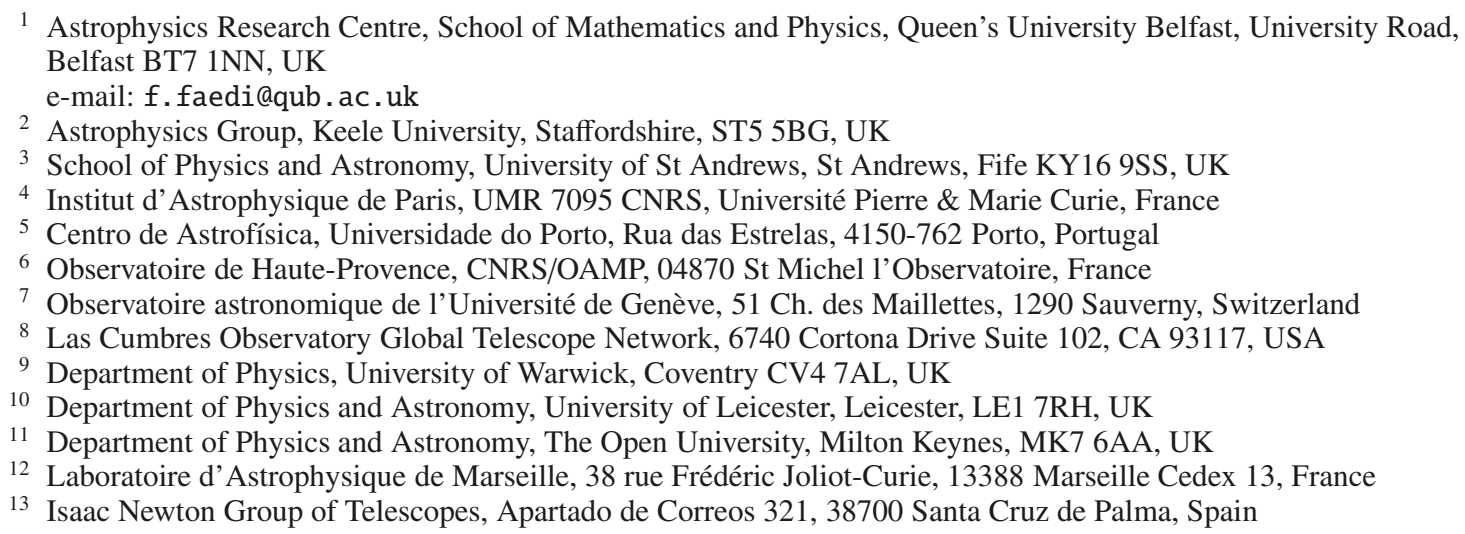

Received 7 February 2011 / Accepted 21 April 2011

\section{ABSTRACT}

\begin{abstract}
We present the discovery of WASP-39b, a highly inflated transiting Saturn-mass planet orbiting a late G-type dwarf star with a period of $4.055259 \pm 0.000008 \mathrm{~d}$, Transit Epoch $T_{0}=2455342.9688 \pm 0.0002$ (HJD), of duration $0.1168 \pm 0.0008 \mathrm{~d}$. A combined analysis of the WASP photometry, high-precision follow-up transit photometry, and radial velocities yield a planetary mass of $M_{\mathrm{pl}}=$ $0.28 \pm 0.03 M_{\mathrm{J}}$ and a radius of $R_{\mathrm{pl}}=1.27 \pm 0.04 R_{\mathrm{J}}$, resulting in a mean density of $0.14 \pm 0.02 \rho_{\mathrm{J}}$. The stellar parameters are mass $M_{\star}=0.93 \pm 0.03 M_{\odot}$, radius $R_{\star}=0.895 \pm 0.23 R_{\odot}$, and age $9_{-4}^{+3}$ Gyr. Only WASP-17b and WASP-31b have lower densities than WASP-39b, although they are slightly more massive and highly irradiated planets. From our spectral analysis, the metallicity of WASP-39 is measured to be $[\mathrm{Fe} / \mathrm{H}]=-0.12 \pm 0.1 \mathrm{dex}$, and we find the planet to have an equilibrium temperature of $1116_{-32}^{+33} \mathrm{~K}$. Both values strengthen the observed empirical correlation between these parameters and the planetary radius for the known transiting Saturn-mass planets.
\end{abstract}

Key words. stars: individual: WASP-39 - techniques: photometric - techniques: radial velocities - planetary systems

\section{Introduction}

The importance of transiting extrasolar planets is related to their geometrical configuration (Sackett 1999). Transit geometry severely constrains the orbital inclination of the planet, allowing accurate measurements of its mass and radius to be derived. The inferred planet's density provides information on the system's bulk physical properties, and thus is a fundamental parameter for constraining theoretical models of planetary formation, structure, and evolution (e.g. Guillot 2005; Fortney et al. 2007; Liu et al. 2008).

To date, more than 100 transiting planets have been discovered, which show a huge range of diversity in their physical and dynamical properties. For example, their mass ranges from

\footnotetext{
* Spectroscopic and photometric data are only available at the CDS via anonymous ftp to

cdsarc.u-strasbg.fr $(130.79 .128 .5)$ or via

http://cdsarc.u-strasbg.fr/viz-bin/qcat? J/A+A/531/A40
}

$\sim 5 M_{\oplus}$ (Kepler-10b, Batalha et al. 2011) to about $12 M_{\mathrm{J}}$ (XO-3b, Johns-Krull et al. 2008; Hébrard et al. 2008). Some planets have radii that agree with models of irradiated planets (Burrows et al. 2007; Fortney et al. 2007), while others are found to be anomalously large (e.g. WASP-12b, Hebb et al. 2009, and TrES-4b, Southworth 2010; Torres et al. 2008; Mandushev et al. 2007). The diversity in exoplanet densities, hence in their internal compositions, is particularly noticeable at sub-Jupiter masses. For example, some exoplanets have very high densities and are thought to have a rocky/ice core (e.g. HD 149026b, $\rho_{\mathrm{pl}} \simeq 1 \rho_{\mathrm{J}}$, Sato et al. 2005), while systems such as TrES-4b $\left(\rho_{\mathrm{pl}}=0.17 \rho_{\mathrm{J}}\right.$, Mandushev et al. 2007), WASP-17b $\left(\rho_{\mathrm{pl}}=0.06 \rho_{\mathrm{J}}\right.$, Anderson et al. 2010b, 2011b), WASP-31b $\left(\rho_{\mathrm{pl}}=0.132 \rho_{\mathrm{J}}\right.$, Anderson et al. 2010a), and Kepler-7b $\left(\rho_{\mathrm{pl}}=0.13 \rho_{\mathrm{J}}\right.$, Latham et al. 2010) are examples of planets with puzzlingly low densities that challenge standard evolutionary theories in reproducing their radii (Fortney et al. 2007; Burrows et al. 2007). To assess the inflation status of a system, generally planetary radii are compared to 
tabulated values from models (e.g. Fortney et al. 2007; Burrows et al. 2007; Baraffe et al. 2008). However, the radius depends on multiple physical properties, such as the stellar age, the irradiation flux, the planet's mass and age, the atmospheric composition, the presence of heavy elements in the envelope or in the core, the atmospheric circulation, and also on any source generating extra heating in the planetary interior. Different mechanisms have been proposed to explain the anomalously large radii, such as tidal heating due to unseen companions pumping up the eccentricity (Bodenheimer et al. 2001, 2003), kinetic heating due to the breaking of atmospheric waves (Guillot \& Showman 2002), enhanced atmospheric opacity (Burrows et al. 2007) and semi-convection (Chabrier \& Baraffe 2007). While each individual mechanism would presumably affect all hot Jupiters to some degree, they cannot explain the entirety of the observed radii (Fortney \& Nettelmann 2010; Baraffe et al. 2010). More complex thermal evolution models are necessary to fully understand their cooling history. For these planets, the dominant source of energy is a function of the orbital separation and the spectral type of the host star, while its dependency on the planetary mass and age is negligible (with the exception of very young planets). More recently, Batygin et al. (2011) performed calculations of the thermal evolution of gas giants and suggest that the extra energy needed to explain the radius inflation comes from stellar irradiation (see also Laughlin et al. 2011). The proposed mechanism (Ohmic heating), based on the interaction of ionised alkali metals in the planetary atmosphere with the planet's magnetic field, along with the atmospheric heavy element content, could provide a universal explanation of the currently measured radius anomalies.

Although the majority of the known exoplanets are shortperiod, Jupiter-mass planets, more recently an increasing number of Saturn-like planets have been discovered (e.g. Enoch et al. 2011), and have encouraged studies of planetary properties and their statistical analysis, searching for possible correlations between planetary parameters (e.g. Enoch et al. 2011; Anderson et al. 2010a; Hartman et al. 2011). To date, 27 transiting planets have been discovered with masses in the range $0.15 M_{\mathrm{J}}<M_{\mathrm{pl}}<0.6 M_{\mathrm{J}}{ }^{1}$, similar to Saturn $\left(M_{\text {Saturn }}=0.229 M_{\mathrm{J}}\right.$, Standish 1995). The detection and characterisation of significantly more bright short-period transiting systems is one of the keys to improving our understanding of planetary structure and evolution.

Here we describe the properties of WASP-39b, a new transiting Saturn-mass planet discovered by the SuperWASP survey. The planet host star WASP-39 belongs to the constellation of Virgo and thus resides in an equatorial region of sky, which is monitored by the SuperWASP-North and WASP-South telescopes simultaneously. WASP-39b is the third least dense planet $\left(\rho_{\mathrm{pl}}=0.14 \pm 0.02 \rho_{\mathrm{J}}\right)$ discovered from a ground-based transit survey, and belongs to the sample of highly inflated gas giant planets. It provides observational evidence for the mass-radius relation of planetary systems in a poorly sampled region of the parameter space.

We present follow-up observations of the new system which establish the planetary nature of the transiting object detected by SuperWASP. High precision, high signal-to-noise lightcurves have been obtained using both the Faulkes Telescope North (FTN), and the Euler telescope, and radial velocity measurements using the SOPHIE (1.93-m OHP) and CORALIE (Swiss 1.2-m) spectrographs.

\footnotetext{
${ }^{1}$ http://exoplanet.eu/
}

Table 1. Photometric properties of WASP-39.

\begin{tabular}{lc}
\hline \hline Parameter & WASP-39 \\
\hline RA(J2000) & $14: 29: 18.42$ \\
Dec(J2000) & $-03: 26: 40.1$ \\
$B$ & $12.93 \pm 0.25$ \\
$V$ & $12.11 \pm 0.13$ \\
$I$ & $11.34 \pm 0.08$ \\
$J$ & $10.663 \pm 0.024$ \\
$H$ & $10.307 \pm 0.023$ \\
$K$ & $10.202 \pm 0.023$ \\
$\mu_{\alpha}(\mathrm{mas} / \mathrm{yr})$ & $-12.2 \pm 3.3$ \\
$\mu_{\delta}(\mathrm{mas} / \mathrm{yr})$ & $2.8 \pm 3.5$ \\
\hline
\end{tabular}

Notes. The broad band magnitudes and proper motion are obtained from the NOMAD 1.0 catalogue.

The paper is structured as follows: in Sect. 2 we describe the observations, including the WASP discovery data and the photometric and spectroscopic follow-up. In Sect. 3 we present our results for the derived systems parameters, and the stellar and planetary properties. Finally, we discuss the implication of the discovery of WASP-39b in Sect. 4.

\section{Observations}

1SWASP J142918.42-032640.1 (2MASS 14291840-0326403), hereafter WASP-39, has been identified in several northern sky catalogues which provide broad-band optical (Zacharias et al. 2005) and infra-red 2MASS magnitudes (Skrutskie et al. 2006) as well as proper motion information. Coordinates, broadband magnitudes, and proper motion of the star are from the NOMAD catalogue and are given in Table 1.

\subsection{SuperWASP observations}

The WASP North and South telescopes are located in La Palma (ING - Canaries Islands) and Sutherland (SAAO South Africa), respectively. Each telescope consists of 8 Canon $200 \mathrm{~mm} f / 1.8$ focal lenses coupled to e $2 \mathrm{v} 2048 \times 2048$ pixel CCDs, yielding a field-of-view of $7.8 \times 7.8$ square degrees with a pixel scale of 13.7" (Pollacco et al. 2006).

WASP-39 is a $V=12.11$ star located in an equatorial region of sky monitored by both WASP instruments, significantly increasing the observing coverage on the target. In January 2009, the SuperWASP-N telescope underwent a system upgrade that improved our control over the main sources of red noise, such as temperature-dependent focus changes (Barros et al. 2011). This upgrade yielded data of unprecedented high quality, and increased the number of planet candidates, flagged in the archive, in particular those with longer period and lower mass (e.g. WASP-38b, Barros et al. 2011; and WASP-39b).

WASP-39 was routinely observed between 2006 July 1 to 2010 July 26, with a total of 11 WASP fields and 40531 photometric points. Over the five WASP seasons only three points were taken in 2006 and none during 2007. However, the same field was observed again in 2008, 2009 and 2010 after both WASP telescopes began observing an overlapping equatorial region.

All data were processed with a custom-built reduction pipeline described in Pollacco et al. (2006). The resulting light curves were analysed using our implementation of the Box Least-Squares and SysRem detrending algorithms (see Collier Cameron et al. 2006; Kovács et al. 2002; Tamuz et al. 2005), 
F. Faedi et al.: WASP-39b: a highly inflated Saturn-mass planet orbitinga late G-type star
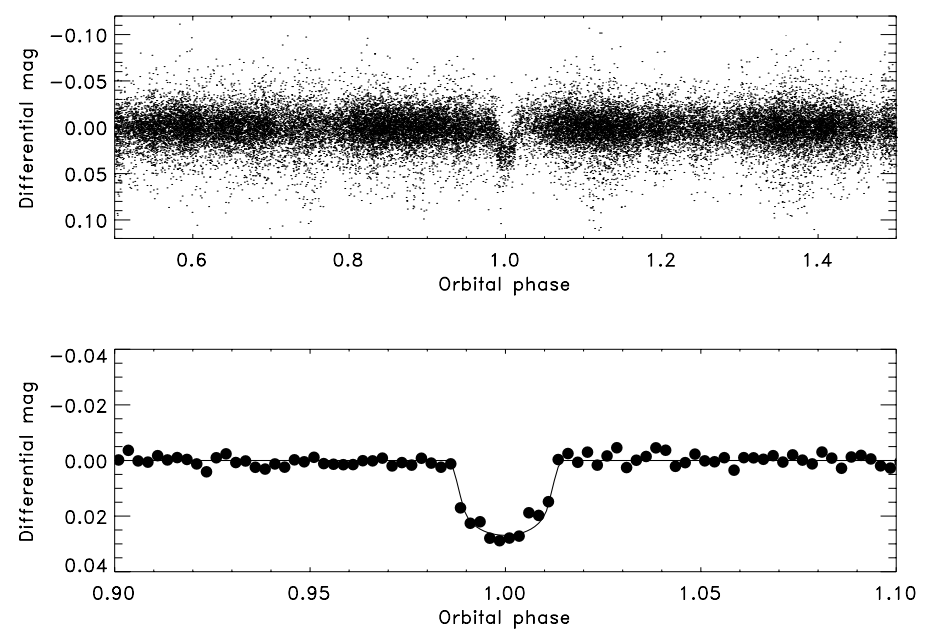

Fig. 1. Upper panel: discovery light curve of WASP-39b phase folded on the ephemeris given in Table 4. Lower panel: binned WASP-39b light curve. Black-solid line, is the best-fit transit model estimated using the formalism from Mandel \& Agol (2002).

to search for signatures of planetary transits. When combined, the SuperWASP light curves showed a characteristic periodic dip in brightness with a period of $P=4.055$ days, duration $T_{14} \sim 168 \mathrm{~min}$, and a depth $\sim 25 \mathrm{mmag}$. Figure 1 shows the discovery photometry of WASP-39b phase folded on the above period, along with the binned phased light curve. A total of 10 partial or full transits were observed, with an improvement in $\chi^{2}$ of the box-shaped model over the flat light curve of $\Delta \chi^{2}=825$. We evaluated a signal-to-(red) noise value of the data following Pont et al. (2006), and determined $S N_{\text {red }}=11.65$.

\subsection{Spectroscopic follow-up}

WASP-39 was first observed during our follow-up campaign in April 2010 at Observatoire de Haute-Provence (OHP). During our program we have obtained follow-up spectroscopy and established the planetary nature of WASP-39b together with three additional systems: WASP-37b (Simpson et al. 2011), WASP38b (Barros et al. 2011) and WASP-40b (Anderson et al. 2011a). Between 2010 April 8 and June 11, we obtained eight radial velocity measurements for WASP-39 using SOPHIE, the fiber-fed echelle spectrograph mounted on the 1.93-m telescope at the OHP (Perruchot et al. 2008; Bouchy et al. 2009). We used SOPHIE in high efficiency mode $(R=40000)$ and obtained observations with very similar signal-to-noise ratio $(\sim 30)$, to minimise systematic errors arising from the known Charge Transfer Inefficiency effect of the CCD (Bouchy et al. 2009), although this is now corrected by the data reduction software. Wavelength calibration with a Thorium-Argon lamp were performed every $\sim 2 \mathrm{~h}$, allowing the interpolation of the spectral drift of SOPHIE ( $<3 \mathrm{~m} \mathrm{~s}^{-1}$ per hour; see Boisse et al. 2010). Two $3^{\prime \prime}$ diameter optical fibres were used, the first centred on the target and the second on the sky to simultaneously measure the background to remove contamination from scattered moonlight. During our observations the contribution from scattered moonlight was negligible as it was well shifted from the target's radial velocity. Nine additional radial velocity measurements were obtained using the CORALIE spectrograph mounted on the 1.2-m Euler Swiss telescope at La Silla, Chile (Baranne et al. 1996; Queloz et al. 2000; Pepe et al. 2002). Observations were obtained with a signal-to-noise of $\sim 30$, during grey/dark
Table 2. Radial velocity (RV) and line bisector span $\left(V_{\text {span }}\right)$ measurements of WASP-39.

\begin{tabular}{cccrl}
\hline \hline $\begin{array}{c}\text { BJD } \\
-2400000\end{array}$ & $\begin{array}{c}\mathrm{RV} \\
\left(\mathrm{km} \mathrm{s}^{-1}\right)\end{array}$ & $\begin{array}{c}\sigma_{\mathrm{RV}} \\
\left(\mathrm{km} \mathrm{s}^{-1}\right)\end{array}$ & $\begin{array}{r}V_{\text {span }} \\
\left(\mathrm{km} \mathrm{s}^{-1}\right)\end{array}$ & Instrument \\
\hline 55321.7484 & -58.455 & 0.011 & -0.017 & CORALIE \\
55327.7544 & -58.523 & 0.022 & -0.047 & CORALIE \\
55359.5932 & -58.511 & 0.011 & -0.067 & CORALIE \\
55361.6552 & -58.502 & 0.035 & -0.128 & CORALIE \\
55362.5676 & -58.471 & 0.013 & -0.050 & CORALIE \\
55365.5935 & -58.455 & 0.012 & -0.048 & CORALIE \\
55376.6394 & -58.534 & 0.012 & -0.071 & CORALIE \\
55378.6265 & -58.473 & 0.009 & -0.036 & CORALIE \\
55380.6376 & -58.522 & 0.012 & 0.006 & CORALIE \\
\hline 55304.4704 & -58.475 & 0.013 & 0.000 & SOPHIE \\
55305.4374 & -58.429 & 0.015 & -0.034 & SOPHIE \\
55323.4389 & -58.565 & 0.017 & -0.031 & SOPHIE \\
55329.4184 & -58.472 & 0.016 & -0.019 & SOPHIE \\
55331.4301 & -58.522 & 0.014 & -0.040 & SOPHIE \\
55334.4624 & -58.418 & 0.017 & -0.036 & SOPHIE \\
55336.4477 & -58.503 & 0.016 & -0.035 & SOPHIE \\
55368.3955 & -58.514 & 0.017 & -0.064 & SOPHIE \\
\hline
\end{tabular}

time to minimise moonlight contamination. The data were processed with the SOPHIE and CORALIE standard data reduction pipelines, respectively. Radial velocity uncertainties were evaluated, including known systematics such as guiding and centring errors (Boisse et al. 2010), and wavelength calibration uncertainties. All spectra were single-lined.

We computed the radial velocities from a weighted crosscorrelation of each spectrum with a numerical mask of spectral type G2, as described in Baranne et al. (1996) and Pepe et al. (2002). The cross-correlation with masks of different spectral types (F0, K5 and M5) produced similar radial velocity variation, rejecting a blended eclipsing system of stars with unequal masses as a possible cause of the variation. Radial velocity measurements and line bisector $\left(V_{\text {span }}\right)$ are given in Table 2, and plotted with the best-fit Keplerian model in Fig. 2. SOPHIE data are plotted as filled circles and CORALIE data as open squares, and both data sets are offset with respect to the radial velocity zero point, $\gamma_{\text {SOPHIE }}$ and $\gamma_{\text {CORALIE }}$, respectively (see Table 4 ). No significant correlation is observed between the radial velocity and the line bisector, suggesting the signal's origin as planetary rather than due to a blended eclipsing binary system or to stellar activity (see Queloz et al. 2001). The rms for SOPHIE and CORALIE radial velocity residuals to the best-fit model are $\mathrm{rms}_{\text {SOPHIE }}=26 \mathrm{~m} \mathrm{~s}^{-1}$ and $\mathrm{rms}_{\text {CORALIE }}=16 \mathrm{~m} \mathrm{~s}^{-1}$, with the higher rms value of the SOPHIE measurements dominated by two discrepant measurements, $R V_{1}=48 \mathrm{~m} \mathrm{~s}^{-1}$ and $R V_{2}=43 \mathrm{~m} \mathrm{~s}^{-1}$. Eliminating the most discrepant of these, the rms SOPHIE becomes $19 \mathrm{~m} \mathrm{~s}^{-1}$, comparable to that of CORALIE. We also plot in Fig. 3 the bisector span measurements as a function of time; and lower panel, the residuals from the RV orbital fit against time. The bisector span values are shifted to a zero mean $\left(\left\langle V_{\text {span }}\right\rangle_{\text {SOPHIE }}=-0.032 \mathrm{~km} \mathrm{~s}^{-1},\left\langle V_{\text {span }}\right\rangle_{\text {CORALIE }}=\right.$ $\left.-0.051 \mathrm{~km} \mathrm{~s}^{-1}\right)$, to better compare the two data sets.

\subsection{Photometric follow-up}

To allow more accurate light curve modelling and thus refine the photometric parameters, we obtained two high signal-tonoise transit light curves of WASP-39b. All photometric data 

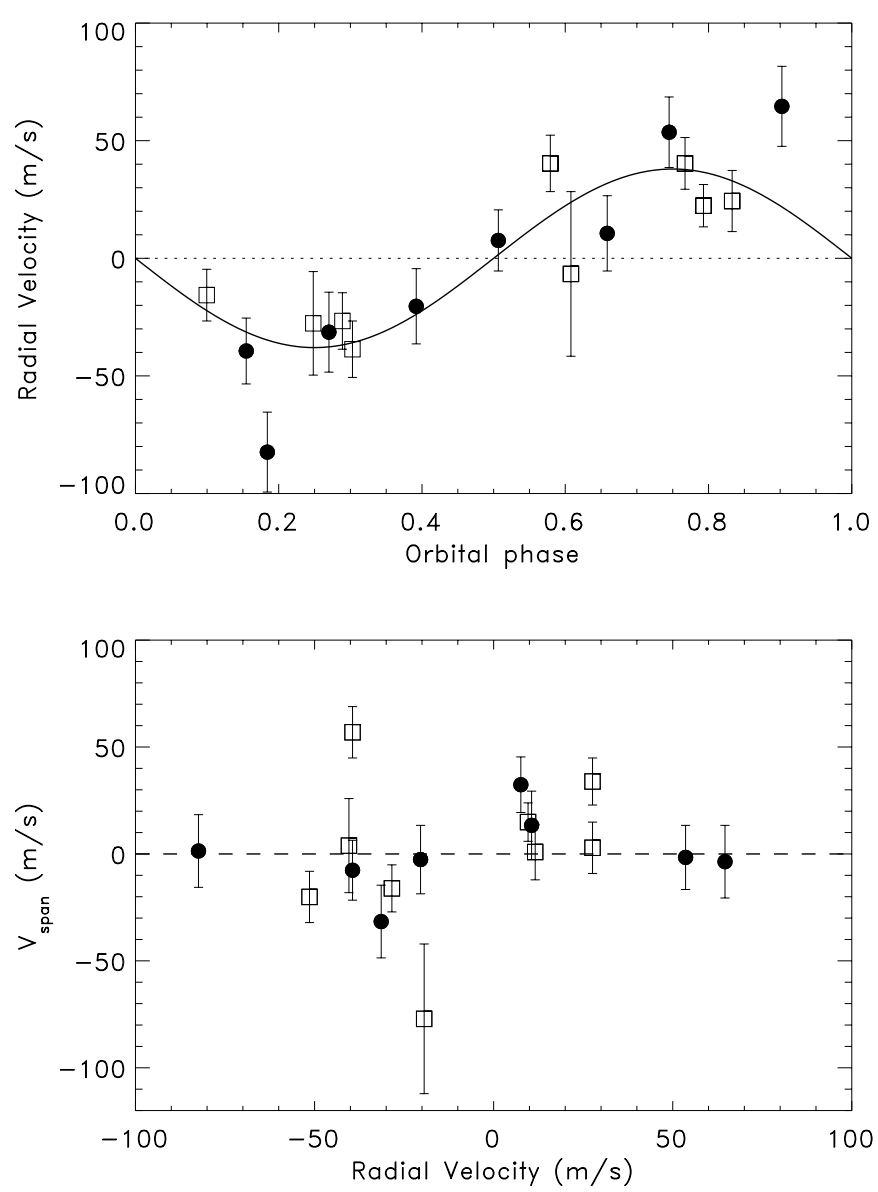

Fig. 2. Upper panel: phase folded radial velocity measurements of WASP-39 obtained combining data from SOPHIE (filled-circles) and CORALIE (open-squares) spectrographs. Superimposed is the best-fit model RV curve with parameters from Table 4 . The centre-of-mass velocity for each data set was subtracted from the RVs $\left(\gamma_{\text {SOPHIE }}=\right.$ $-58.4826 \mathrm{~km} \mathrm{~s}^{-1}$ and $\gamma_{\text {CORALIE }}=-58.4708 \mathrm{~km} \mathrm{~s}^{-1}$ ). Lower panel: we show the bisector span measurements as a function of radial velocity, values are shifted to a zero-mean $\left(\left\langle V_{\text {span }}\right\rangle_{\text {SOPHIE }}=-0.032 \mathrm{~km} \mathrm{~s}^{-1}\right.$, $\left.\left\langle V_{\text {span }}\right\rangle_{\text {CORALIE }}=-0.051 \mathrm{~km} \mathrm{~s}^{-1}\right)$. The bisector span shows no significant variation nor correlation with the RVs, suggesting that the signal is mainly due to Doppler shifts of the stellar lines rather than stellar profile variations due to stellar activity or a blended eclipsing binary.

presented here are available from the NStED database ${ }^{2}$. The first full transit was observed with the LCOGT $^{3} 2 \mathrm{~m}^{\text {Faulkes }}$ Telescope North (FTN) on Haleakala, Maui Hawai'i, on the night of 2010 May 18. The fs03 Spectral Instruments camera was used with a $2 \times 2$ binning mode giving a field-of-view of $10^{\prime} \times 10^{\prime}$ and a pixel scale of $0.303^{\prime \prime} /$ pixel. Data were taken through a Pan-STARRS- $z$ filter and the telescope was defocussed during observations to prevent saturation and allow longer exposure times to be used. The observations were pre-processed using the WASP Pipeline (Pollacco et al. 2006) to perform master bias and flat construction, debiassing and flatfielding. Aperture photometry was performed with the the DAOPHOT package (Stetson 1987) within the $\mathrm{IRAF}^{4}$ environment using a 13 pixel

\footnotetext{
${ }^{2}$ http://nsted.ipac.caltech.edu

${ }^{3}$ http://lcogt.net

${ }^{4}$ IRAF is distributed by the National Optical Astronomy Observatory, which is operated by the Association of Universities for Research in Astronomy, Inc., under cooperative agreement with the National Science Foundation.
}
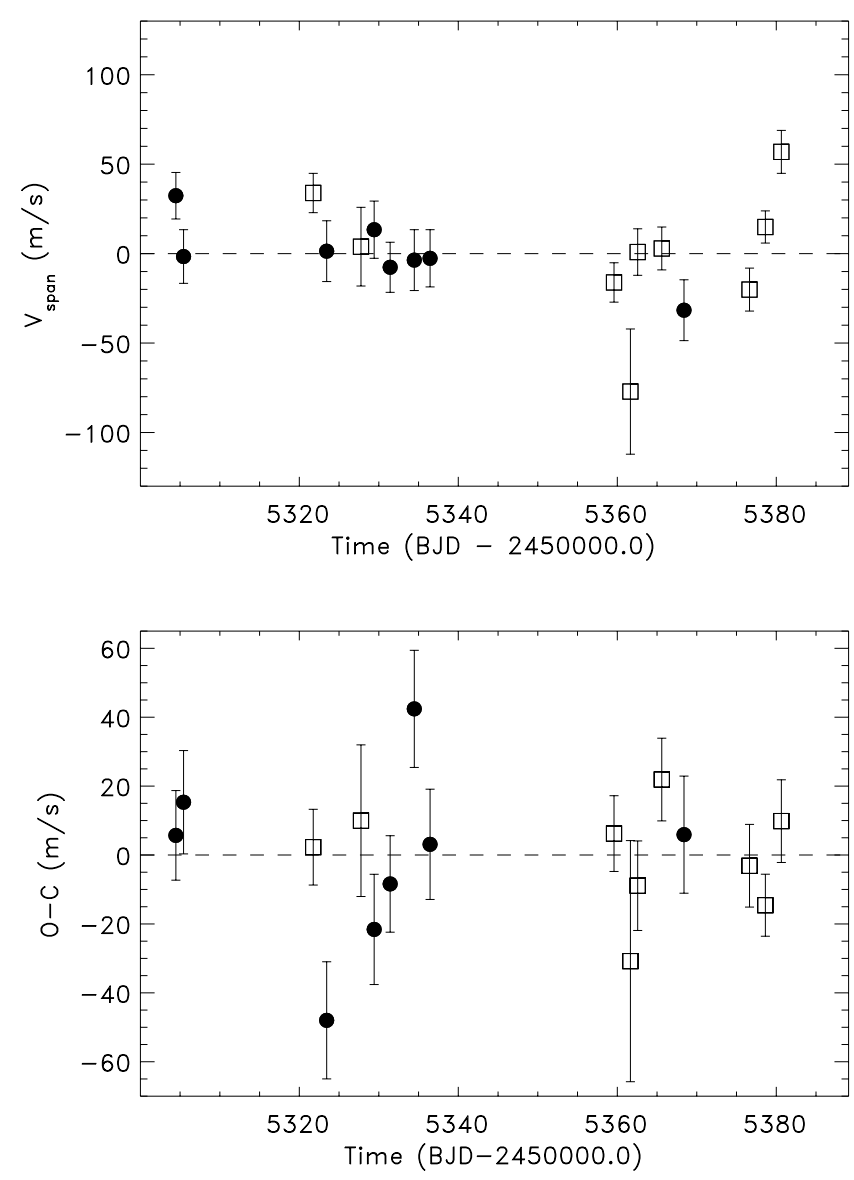

Fig. 3. Upper panel: the bisector span measurements as a function of time (BJD-2450000.0), $V_{\text {span }}$ values are shifted to a zero-mean as in Fig. 2. Lower panel: residuals from the RV orbital fit plotted against time.

radius aperture. The differential photometry was performed relative to 7 comparison stars that were within the FTN field of view. Additional high signal-to-noise photometry was obtained in the Gunn $r$ filter with the Euler-Swiss telescope on 2010 July 9, only covering a partial transit. Conditions were variable, with seeing ranging from $0.6^{\prime \prime}$ to $1.7^{\prime \prime}$. The Euler telescope employs an absolute tracking system, which keeps the star on the same pixel during the observation by matching the point sources in each image with a catalogue, and adjusting the telescope pointing between exposures to compensate for drifts. Observations were obtained with a slightly defocused $(0.1 \mathrm{~mm})$ telescope. All images were corrected for bias and flat field effects and the light curve was obtained by performing relative aperture photometry of the target and one bright reference star.

Both the FTN and Euler light curves are shown in Fig. 4. The photometry confirms the transit which phases with the ephemeris derived from the WASP discovery photometry.

\section{Results}

\subsection{Stellar parameters}

A total of nine individual CORALIE spectra of WASP-39 were co-added to produce a single spectrum with a typical $S / N$ ratio of around 50. The standard pipeline reduction products were used in the analysis. To improve the line profile fitting for equivalent width measurements, the spectrum was smoothed using a 


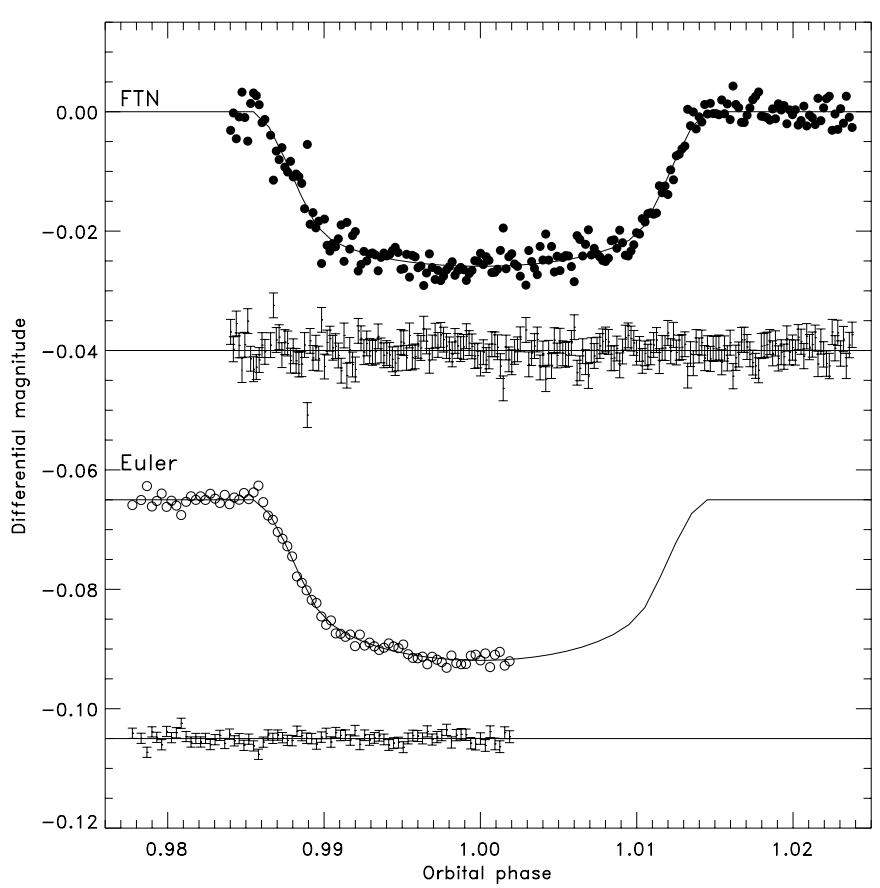

Fig. 4. FTN $z$-band and Euler $r$-band follow-up high signal-to-noise photometry of WASP-39b during the transit. The Euler light curve has been ofsetted from zero by an arbitrary amount for clarity. The data are phase-folded on the ephemeris from Table 4. Superposed (black-solid line) is the best-fit transit model estimated using the formalism from Mandel \& Agol (2002). Residuals from the fit are displayed underneath.

Gaussian width $\sigma=0.05 \AA$. For the $v \sin i^{\star}$ determination the unsmoothed spectrum was used.

Our analysis was performed using the methods given in Gillon et al. (2009). The $\mathrm{H}_{\alpha}$ line was used to determine the effective temperature $\left(T_{\text {eff }}\right)$, while the $\mathrm{Na} \mathrm{I} \mathrm{D}$ and $\mathrm{Mg}$ I $\mathrm{b}$ lines were used as surface gravity $(\log g)$ diagnostics. The atmospheric parameters obtained from the analysis are listed in Table 3. The elemental abundances were determined from equivalent width measurements of several clean and unblended lines. A value for microturbulence $\left(\xi_{\mathrm{t}}\right)$ was determined from the $\mathrm{Fe} \mathrm{I}$ lines using the method of Magain (1984). Quoted error estimates include those given by the uncertainties in $T_{\text {eff }}, \log g$ and $\xi_{\mathrm{t}}$, as well as the scatter due to measurement and atomic data uncertainties.

The projected stellar rotation velocity $\left(v \sin i^{\star}\right)$ was determined by fitting the profiles of several unblended $\mathrm{Fe}$ I lines. A value for macroturbulence $\left(v_{\mathrm{mac}}\right)$ of $2.1 \pm 0.3 \mathrm{~km} \mathrm{~s}^{-1}$ was assumed, based on the tabulation by Gray (2008) and an instrumental $F W H M$ of $0.11 \pm 0.01 \AA$, determined from the telluric lines around $6300 \AA$. A best-fitting value of $v \sin i^{\star}=1.4 \pm$ $0.6 \mathrm{~km} \mathrm{~s}^{-1}$ was obtained. The stellar mass $M_{\star}$ and radius $R_{\star}$ were estimated using the calibration of Torres et al. (2010).

The non-detection of lithium in the spectrum, the low rotation rate implied by the $v \sin i^{\star}$ and lack of stellar activity (shown by the absence of $\mathrm{Ca}$ II $\mathrm{H}$ and $\mathrm{K}$ emission) all indicate that the star is relatively old. Unfortunately, the gyrochronological age estimate from the Barnes (2007) relation $\left(\sim 5_{-4}^{+20} \mathrm{Gyr}\right)$ can only provide a weak constraint on the age of WASP-39.

The stellar density of $\rho_{\star}=1.297_{-0.074}^{+0.080} \rho_{\mathrm{J}}$ obtained from the MCMC analysis was used together with the stellar temperature and metallicity values, derived from spectroscopy, in an interpolation of the Yonsei-Yale stellar evolution tracks (Demarque et al. 2004), as shown in Fig. 5. Using the best-fit metallicity of $[\mathrm{Fe} / \mathrm{H}]=-0.12$, we obtain a mass for WASP-39 of
Table 3. Stellar parameters of WASP-39 from spectroscopic analysis.

\begin{tabular}{lc}
\hline \hline Parameter & Value \\
\hline$T_{\text {eff }}$ & $5400 \pm 150 \mathrm{~K}$ \\
$\log g$ & $4.4 \pm 0.2$ \\
$\xi_{\mathrm{t}}$ & $0.9 \pm 0.2 \mathrm{~km} \mathrm{~s}^{-1}$ \\
$v \sin i^{\star}$ & $1.4 \pm 0.6 \mathrm{~km} \mathrm{~s}^{-1}$ \\
{$[\mathrm{Fe} / \mathrm{H}]$} & $-0.12 \pm 0.10$ \\
{$[\mathrm{Na} / \mathrm{H}]$} & $-0.04 \pm 0.10$ \\
{$[\mathrm{Mg} / \mathrm{H}]$} & $0.06 \pm 0.11$ \\
{$[\mathrm{Al} / \mathrm{H}]$} & $0.01 \pm 0.08$ \\
{$[\mathrm{Si} / \mathrm{H}]$} & $0.04 \pm 0.08$ \\
{$[\mathrm{Ca} / \mathrm{H}]$} & $0.01 \pm 0.14$ \\
{$[\mathrm{Sc} / \mathrm{H}]$} & $0.02 \pm 0.19$ \\
{$[\mathrm{Ti} / \mathrm{H}]$} & $-0.03 \pm 0.10$ \\
{$[\mathrm{~V} / \mathrm{H}]$} & $-0.08 \pm 0.17$ \\
{$[\mathrm{Cr} / \mathrm{H}]$} & $-0.07 \pm 0.10$ \\
{$[\mathrm{Mn} / \mathrm{H}]$} & $-0.03 \pm 0.20$ \\
{$[\mathrm{Co} / \mathrm{H}]$} & $-0.10 \pm 0.12$ \\
{$[\mathrm{Ni} / \mathrm{H}]$} & $-0.06 \pm 0.09$ \\
$\log A(\mathrm{Li})$ & $<0.9$ \\
$\mathrm{Mass}$ & $0.93 \pm 0.09 M_{\odot}$ \\
$\mathrm{Radius}$ & $1.00 \pm 0.25 R_{\odot}$ \\
$\mathrm{Sp} . \mathrm{Type}$ & $\mathrm{G} 8$ \\
$\mathrm{Distance}$ & $230 \pm 80 \mathrm{pc}$ \\
\hline
\end{tabular}

Notes. Mass and Radius estimate using the Torres et al. (2010) calibration. Spectral Type estimated from $T_{\text {eff }}$ using the table in Gray (2008).

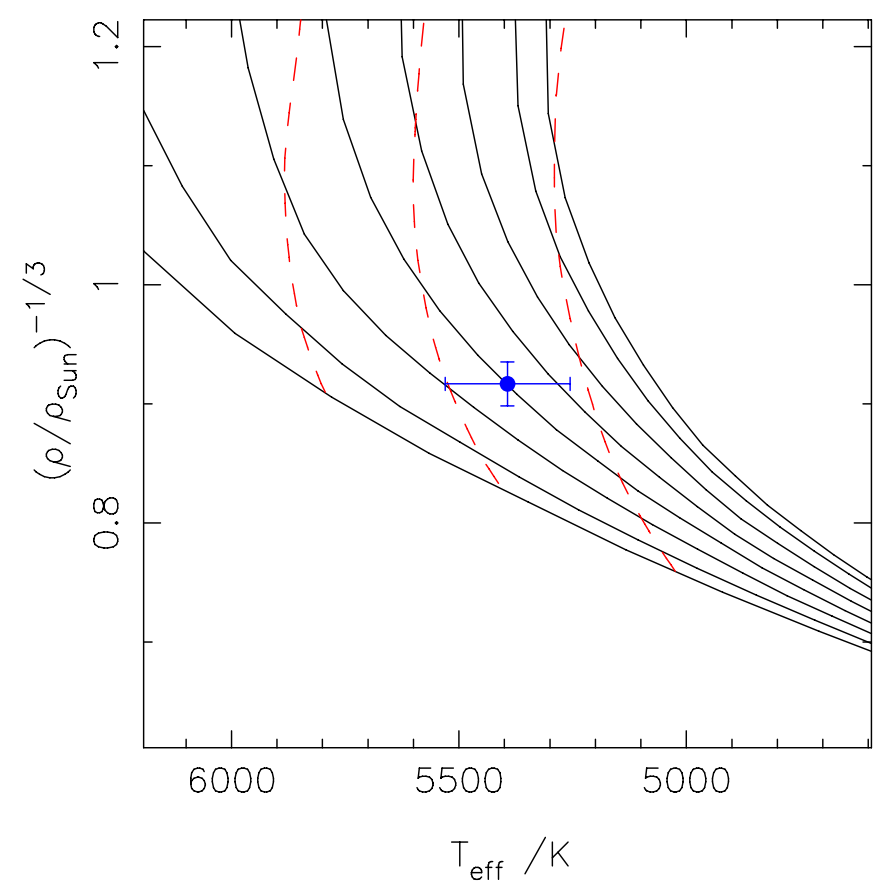

Fig. 5. Isochrone tracks from Demarque et al. (2004) for WASP-39 using the best-fit metallicity of $[\mathrm{Fe} / \mathrm{H}]=-0.12 \mathrm{dex}$ and stellar density $1.297 \rho_{\odot}$. Solid lines are for isochrones of, left to right: 1.0, 3.0, 6.0, $9.0,12.0,15.0,18.0$, and $20.0 \mathrm{Gyr}$. Dashed lines are for mass tracks of, left to right: $1.0,0.9,0.8 \mathrm{M}_{\odot}$.

$0.86 \pm 0.05 M_{\odot}$ and a stellar age of $9_{-4}^{+3} \mathrm{Gyr}$, in agreement with the gyrochronological age and a more accurate estimate. The mass obtained from the YY-isochrone fit is somewhat less than the value from the Torres et al. (2010) calibration (as was also found in the analysis of WASP-37 stellar parameters; Simpson et al. 2011), but their 1- $\sigma$ errors overlap. 


\subsection{Planetary parameters}

The planetary properties were determined using a simultaneous Markov-Chain Monte Carlo (MCMC) analysis including the WASP photometry, the follow-up FTN and Euler photometry, together with SOPHIE and CORALIE radial velocity measurements. A detailed description of the method is given in Collier Cameron et al. (2007) and Pollacco et al. (2008). The parameters we used in the fit are: the epoch of mid-transit $T_{0}$, the orbital period $P$, the fractional change of flux proportional to the ratio of stellar to planet surface areas $\Delta F=R_{\mathrm{pl}}^{2} / R_{\star}^{2}$, the transit duration $T_{14}$, the impact parameter $b$, the radial velocity semi-amplitude $K_{1}$, the stellar effective temperature $T_{\text {eff }}$, metallicity $[\mathrm{Fe} / \mathrm{H}]$, the Lagrangian elements $\sqrt{e} \cos \omega$ and $\sqrt{e} \sin \omega$ (where $e$ is the eccentricity and $\omega$ the longitude of periastron), and the systematic offset velocity $\gamma$. In this particular case we fitted the 2 systematic velocities $\gamma_{\text {SOPHIE }}$ and $\gamma_{\text {CORALIE }}$ to allow for instrumental offsets between the two datasets.

Four different sets of solutions were considered: with and without the main-sequence mass-radius constraint in the case of circular orbits and orbits with floating eccentricity. For each solution we have included a linear trend in the systemic velocity, as a free parameter. However, we find no significant variation. We used the model of Claret $(2000,2004)$ for the limb-darkening in the $r$-band, for both WASP and Euler photometry, and in the $z$-band for FTN photometry. Due to the low mass of WASP-39b, the radial velocity data do not offer convincing evidence for an eccentric orbit. We performed a Lucy \& Sweeney (1971, Eq. (27)) F-test, which indicates that there is a 54\% probability that the improvement in the fit produced by the best-fitting eccentricity could have arisen by chance if the orbit were truly circular. Moreover, we find that imposing the main-sequence constraint has little effect on the MCMC global solution. Thus, we decided to adopt no main-sequence prior and circular orbit.

From the above parameters, we calculate the mass $M$, radius $R$, density $\rho$, and surface gravity $\log g$ of the star (which we denote with subscript $\star$ ) and the planet (which we denote with subscript pl), as well as the equilibrium temperature of the planet assuming it to be a black-body $T_{\mathrm{pl}, \mathrm{A}=0}$ and that energy is efficiently redistributed from the planet's day-side to its nightside. We also calculate the transit ingress/egress times $T_{12} / T_{34}$, and the orbital semi-major axis $a$. These calculated values and their 1- $\sigma$ uncertainties from our MCMC analysis are presented in Table 4. The corresponding best-fitting transit light curves are shown in Figs. 1, 4, and the best-fitting RV curve in Fig. 2.

\section{Discussion}

We report the discovery of a new transiting extrasolar planet, WASP-39b. A simultaneous fit to transit photometry and radial velocity measurements gives a planetary mass of $0.28 \pm$ $0.03 M_{\mathrm{J}}$ and a radius of $1.27 \pm 0.040 R_{\mathrm{J}}$ which yields a planetary density of $0.141 \pm 0.02 \rho_{\mathrm{J}}$. Thus, WASP-39b is the third least dense planet identified by a ground-based transit survey. Only WASP-17b (Anderson et al. 2010b, $\rho_{W 17}=0.06 \rho_{\mathrm{J}}$ ), and WASP-31b $\left(\rho_{W 31}=0.132 \rho_{\mathrm{J}}\right.$, Anderson et al. 2010a) have lower densities. However, they are slightly more massive planets $\left(M_{W 17}=0.49 M_{\mathrm{J}}\right.$ and $\left.M_{W 31}=0.48 M_{\mathrm{J}}\right)$, but highly irradiated, with larger and hotter host stars (Anderson et al. 2010b,a). This implies higher planet equilibrium temperatures for WASP$17 \mathrm{~b}$ and WASP-31b compared to WASP-39b. We find WASP$39 \mathrm{~b}$ to have a highly inflated radius $\left(R_{\mathrm{pl}}=1.27 R_{\mathrm{J}}\right)$, more than $20 \%$ larger than the $R_{\mathrm{pl}}$ obtained by comparison with the Fortney et al. (2007) and the Baraffe et al. (2008) models for a coreless
Table 4. System parameters of WASP-39.

\begin{tabular}{lc}
\hline \hline Parameter (unit) & Value \\
\hline$P(\mathrm{~d})$ & $4.055259 \pm 0.000009$ \\
$T_{0}(\mathrm{HJD})$ & $2455342.9688 \pm 0.0002$ \\
$T_{14}(\mathrm{~d})^{a}$ & $0.1168 \pm 0.0008$ \\
$T_{12}=T_{34}(\mathrm{~d})$ & $0.0179 \pm 0.0009$ \\
$\Delta F=R_{\mathrm{pl}}^{2} / R_{\star}^{2}$ & $0.0211_{-0.0004}^{+0.0003}$ \\
$b$ & $0.441_{-0.043}^{+0.036}$ \\
$i\left(^{\circ}\right)$ & $87.83_{-0.22}^{+0.25}$ \\
$K_{1}\left(\mathrm{~m} \mathrm{~s}^{-1}\right)$ & $38 \pm 4$ \\
$\gamma_{\mathrm{SOPHIE}}\left(\mathrm{km} \mathrm{s}^{-1}\right)$ & $-58.4826 \pm 0.0004$ \\
$\gamma_{\mathrm{CORALIE}}\left(\mathrm{km} \mathrm{s}^{-1}\right)$ & $-58.4708 \pm 0.0004$ \\
$e$ & $0(\mathrm{fixed})$ \\
$M_{\star}\left(M_{\odot}\right)$ & $0.93 \pm 0.03$ \\
$R_{\star}\left(R_{\odot}\right)$ & $0.895 \pm 0.023$ \\
$\log g_{\star}(\mathrm{cgs})$ & $4.503 \pm 0.017$ \\
$\rho_{\star}\left(\rho_{\odot}\right)$ & $1.297_{-0.074}^{+0.082}$ \\
$M_{\mathrm{pl}}\left(M_{\mathrm{J}}\right)$ & $0.28 \pm 0.03$ \\
$R_{\mathrm{pl}}\left(R_{\mathrm{J}}\right)$ & $1.27 \pm 0.04$ \\
$\log g_{\mathrm{pl}}(\mathrm{cgs})$ & $2.610_{-0.053}^{+0.047}$ \\
$\rho_{\mathrm{pl}}\left(\rho_{\mathrm{J}}\right)$ & $0.14 \pm 0.02$ \\
$a(\mathrm{AU})$ & $0.0486 \pm 0.0005$ \\
$T_{\mathrm{pl}, \mathrm{A}=0}(\mathrm{~K})$ & $1116_{-32}^{+33}$ \\
\hline &
\end{tabular}

Notes. ${ }^{(a)} T_{14}$ : time between 1 st and 4 th contact.

planet of a similar mass, orbital distance and stellar age. For example, tables presented in Fortney et al. (2007) predict a maximum radius of $\sim 1.05 R_{\mathrm{J}}$ for a $0.24 M_{\mathrm{J}}$ planet orbiting at $0.045 \mathrm{AU}$ from a $4.5 \mathrm{Gyr}$ solar-type star. In addition, WASP-39 is smaller, cooler and probably older than the Sun. Thus, a radius of $1.27 R_{\mathrm{J}}$ is clearly too large for these models. The fact that we do not detect any eccentricity, and that the age of WASP39b's host star is $\sim 9$ Gyr, suggests that it is unlikely that recent tidal circularisation and dissipation could be a cause of the large radius of WASP-39b (Leconte et al. 2010; Hansen 2010). The low metallicity $([\mathrm{Fe} / \mathrm{H}]=-0.12 \pm 0.1)$ of the WASP-39b host star supports the expected low planetary core-mass. However, this will only marginally explain the highly inflated radius of WASP-39b. Hence, this leads to the hypothesis that some additional physics is at play (Fortney et al. 2010). An interior heat source, replacing the radiated heat from gravitational contraction, is needed for the planet to reach thermal equilibrium with a larger radius than theoretically expected. The Ohmic heating mechanism (Batygin et al. 2011), based on the electro-magnetic interaction betweent atmospheric winds and the planet's magnetic field, is able to explain the anomalous sizes of close-in transiting planets. WASP-39b has a low equilibrium temperature $\left(T_{\mathrm{pl}, \mathrm{A}=0}=1116 \mathrm{~K}\right)$ and thus appears to belong to the "pL" class of planets from Fortney et al. (2008). We note that, with the measured planetary parameters (mass, radius, $T_{\mathrm{eq}}$ ), WASP-39b agrees with the coreless models in Fig. 4 of Batygin et al. (2011).

Of the known transiting systems, WASP-39b joins an increasing number of recently discovered exoplanets with Saturn-like masses. With an increasingly large sample of wellcharacterised systems, we can begin to make statistical inferences as to the physical reasons behind their diverse nature. Enoch et al. (2011) and Anderson et al. (in prep.), show that the radii of known low-mass $\left(0.15-0.6 M_{\mathrm{J}}\right)$ giant planets strongly 

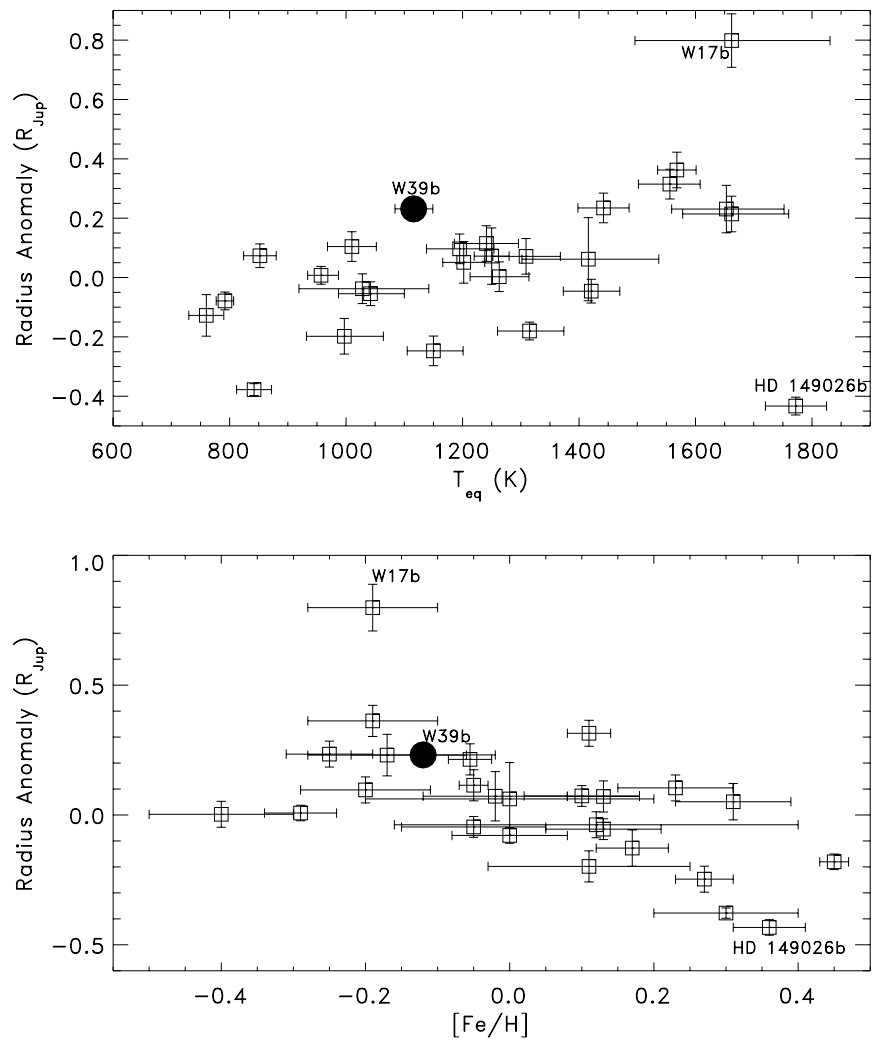

Fig. 6. Upper panel: the radius anomaly $\mathcal{R}=R_{\text {obs }}-R_{\text {pred }}$, versus equilibrium temperature for the known Saturn-mass planets. Lower panel: $\mathcal{R}$ as function of the stellar metallicity $[\mathrm{Fe} / \mathrm{H}]$ in dex. WASP-39b is indicated with a filled circle.

correlate with equilibrium temperature and host-star metallicity. We have investigated how WASP-39b relates to the rest of the sample of Saturn-mass planets. We calculated the $\mathrm{ra}$ dius anomaly $\mathcal{R}$, as in Laughlin et al. (2011), for all Saturnmass planets included in the list from Enoch et al. (2011), and added the latest discoveries (for an updated list see the extrasolar planet encyclopedia http://exoplanet.eu/). In Fig. 6 we plot $\mathcal{R}$ against the planetary equilibrium temperature (upper panel), and as a function of the host star metallicity (lower panel). WASP-39b is indicated with a filled circle. Figure 6 shows that $\mathcal{R}$ correlates with the equilibrium temperature of the planet, as also found by Laughlin et al. (2011). For transiting gas giant planets the dominant source of energy is a function of the orbital separation and the spectral type of the host star, and depends negligibly on the planetary mass and age (with the exception of very young planets). Thus, the observed $\mathcal{R}-T_{\text {eq }}$ trend supports the relation between the inflation mechanism and the stellar irradiation flux (Laughlin et al. 2011). The masses of planets in the Saturn-mass range also appear to correlate with their host star metallicity (Guillot et al. 2006; Burrows et al. 2007), such that low-density planets are found to orbit sub-solar metallicity stars (for example WASP-21 $[\mathrm{Fe} / \mathrm{H}]=-0.4$, Bouchy et al. 2010), while higher density planets orbit stars with super-solar metallicity (for example WASP-29 $[\mathrm{Fe} / \mathrm{H}]=0.11 \mathrm{dex}$, Hellier et al. 2010, HD $149026[\mathrm{Fe} / \mathrm{H}]=0.36 \mathrm{dex}$, Sato et al. 2005, and Kepler-9 $[\mathrm{Fe} / \mathrm{H}]=0.12 \mathrm{dex}$, Holman et al. 2010). Figure 6 shows the observed trend between $\mathcal{R}-[\mathrm{Fe} / \mathrm{H}]$, and highlights the large diversity of planetary internal structure and composition. WASP-39's metallicity $([\mathrm{Fe} / \mathrm{H}]=-0.12$ dex $)$ strengthens this correlation possibly supporting the core-accretion scenario for planet formation (Guillot et al. 2006; Hartman et al. 2009;
Bouchy et al. 2010). The Ohmic dissipation mechanism (Batygin et al. 2011), coupled to the diversity of heavy element abundances (planetary structural evolution), could provide a universal mechanism able to slow down planetary cooling and thus the shrinking of the radius, possibly explaining the observed correlation between planetary radii and equilibrium temperature. Further progress may be achieved with a better understanding of the structure and atmospheric composition of transiting planets (clouds, chemistry, etc.).

Acknowledgements. The SuperWASP Consortium consists of astronomers primarily from Queens University Belfast, St Andrews, Keele, Leicester, The Open University, Isaac Newton Group La Palma and Instituto de Astrofsica de Canarias. The SuperWASP-N camera is hosted by the Issac Newton Group on La Palma and WASPSouth is hosted by SAAO. We are grateful for their support and assistance. Funding for WASP comes from consortium universities and from the UK's Science and Technology Facilities Council. F.P.K. is grateful to AWE Aldermaston for the award of a William Penney Fellowship. Based on observations made with SOPHIE spectrograph mounted on the 1.9-m telescope at Observatoire de Haute-Provence (CNRS), France and at the ESO La Silla Observatory (Chile) with the CORALIE Echelle spectrograph mounted on the Swiss telescope. The research leading to these results has received funding from the European Community's Seventh Framework Programme (FP7/2007-2013) under grant agreement number RG226604 (OPTICON). F.F. is grateful to Yilen Gómez Maqueo Chew for proofreading this paper. F.F. is grateful to the anonymous referee for useful comments improving the paper.

\section{References}

Anderson, D. R., Collier Cameron, A., Hellier, C., et al. 2010a, ApJ, 709, 159 Anderson, D. R., Hellier, C., Gillon, M., et al. 2010b [arXiv: 1011.5882] Anderson, D. R., Barros, S. C. C., Boisse, I., et al. 2011a [arXiv: 1101.5620] Anderson, D. R., Smith, A. M. S., Lanotte, A. A., et al. $2011 \mathrm{~b}$ [arXiv: 1101.4643]

Baraffe, I., Chabrier, G., \& Barman, T. 2008, A\&A, 482, 315

Baraffe, I., Chabrier, G., \& Barman, T. 2010, Rep. Prog. Phys., 73, 016901

Baranne, A., Queloz, D., Mayor, M., et al. 1996, A\&AS, 119, 373

Barnes, S. A. 2007, ApJ, 669, 1167

Barros, S. C. C., Faedi, F., Collier Cameron, A., et al. 2011, A\&A, 525, A54

Batalha, N. M., Borucki, W. J., Bryson, S. T., et al. 2011, ApJ, 729, 27

Batygin, K., Stevenson, D. J., \& Bodenheimer, P. H. 2011 [arXiv: 1101.3800]

Bodenheimer, P., Lin, D. N. C., \& Mardling, R. A. 2001, ApJ, 548, 466

Bodenheimer, P., Laughlin, G., \& Lin, D. N. C. 2003, ApJ, 592, 555

Boisse, I., Eggenberger, A., Santos, N. C., et al. 2010, A\&A, 523, A88

Bouchy, F., Hébrard, G., Udry, S., et al. 2009, A\&A, 505, 853

Bouchy, F., Hebb, L., Skillen, I., et al. 2010, A\&A, 519, A98

Burrows, A., Hubeny, I., Budaj, J., \& Hubbard, W. B. 2007, ApJ, 661, 502

Chabrier, G., \& Baraffe, I. 2007, ApJ, 661, L81

Claret, A. 2000, A\&A, 363, 1081

Claret, A. 2004, A\&A, 428, 1001

Collier Cameron, A., Pollacco, D., Street, R. A., et al. 2006, MNRAS, 373, 799

Collier Cameron, A., Wilson, D. M., West, R. G., et al. 2007, MNRAS, 380, 1230

Demarque, P., Woo, J., Kim, Y., \& Yi, S. K. 2004, ApJ, 155, 667

Enoch, B., Cameron, A. C., Anderson, D. R., et al. 2011, MNRAS, 410, 1631

Fortney, J. J., \& Nettelmann, N. 2010, Space Sci. Rev., 152, 423

Fortney, J. J., Marley, M. S., \& Barnes, J. W. 2007, ApJ, 659, 1661

Fortney, J. J., Lodders, K., Marley, M. S., \& Freedman, R. S. 2008, ApJ, 678, 1419

Fortney, J. J., Baraffe, I., \& Militzer, B. 2010, in Exoplanets, ed. S. Seager (Tucson, AZ: University of Arizona Press), 397

Gillon, M., Smalley, B., Hebb, L., et al. 2009, A\&A, 496, 259

Gray, D. F. 2008, The Observation and Analysis of Stellar Photospheres, ed. D. F. Gray

Guillot, T. 2005, Ann. Rev. Earth Plan. Sci., 33, 493

Guillot, T., \& Showman, A. P. 2002, A\&A, 385, 156

Guillot, T., Santos, N. C., Pont, F., et al. 2006, A\&A, 453, L21

Hansen, B. M. S. 2010, ApJ, 723, 285

Hartman, J. D., Bakos, G. Á., Torres, G., et al. 2009, ApJ, 706, 785

Hartman, J. D., Bakos, G. Á., Sato, B., et al. 2011, ApJ, 726, 52

Hebb, L., Collier-Cameron, A., Loeillet, B., et al. 2009, ApJ, 693, 1920

Hébrard, G., Bouchy, F., Pont, F., et al. 2008, A\&A, 488, 763

Hellier, C., Anderson, D. R., Collier Cameron, A., et al. 2010, ApJ, 723, L60

Holman, M. J., Fabrycky, D. C., Ragozzine, D., et al. 2010, Science, 330, 51

Johns-Krull, C. M., McCullough, P. R., Burke, C. J., et al. 2008, ApJ, 677, 657

Kovács, G., Zucker, S., \& Mazeh, T. 2002, A\&A, 391, 369 
Latham, D. W., Borucki, W. J., Koch, D. G., et al. 2010, ApJ, 713, L140 Laughlin, G., Crismani, M., \& Adams, F. C. 2011, ApJ, 729, L7

Leconte, J., Chabrier, G., Baraffe, I., \& Levrard, B. 2010, A\&A, 516, A64

Liu, X., Burrows, A., \& Ibgui, L. 2008, ApJ, 687, 1191

Lucy, L. B., \& Sweeney, M. A. 1971, AJ, 76, 544

Magain, P. 1984, A\&A, 134, 189

Mandel, K., \& Agol, E. 2002, ApJ, 580, L171

Mandushev, G., O'Donovan, F. T., Charbonneau, D., et al. 2007, ApJ, 667, L195

Pepe, F., Mayor, M., Galland, F., et al. 2002, A\&A, 388, 632

Pollacco, D. L., Skillen, I., Collier Cameron, A., et al. 2006, PASP, 118, 1407

Perruchot, S., Kohler, D., Bouchy, F., et al. 2008, in SPIE Conf. Ser., 7014

Pollacco, D., Skillen, I., Collier Cameron, A., et al. 2008, MNRAS, 385, 1576

Pont, F., Zucker, S., \& Queloz, D. 2006, MNRAS, 373, 231

Queloz, D., Eggenberger, A., Mayor, M., et al. 2000, A\&A, 359, L13
Queloz, D., Henry, G. W., Sivan, J. P., et al. 2001, A\&A, 379, 279

Sackett, P. D. 1999, in Planets Outside the Solar System: Theory and Observations, ed. J-M. Mariotti, \& D. Alloin, NATO ASIC Proc. 532, 189

Sato, B., Fischer, D. A., Henry, G. W., et al. 2005, ApJ, 633, 465

Simpson, E. K., Faedi, F., Barros, S. C. C., et al. 2011, AJ, 141, 8

Skrutskie, M. F., Cutri, R. M., Stiening, R., et al. 2006, AJ, 131, 1163

Southworth, J. 2010, MNRAS, 408, 1689

Standish, E. M. 1995, Highlights of Astronomy, 10, 180

Stetson, P. B. 1987, PASP, 99, 191

Tamuz, O., Mazeh, T., \& Zucker, S. 2005, MNRAS, 356, 1466

Torres, G., Winn, J. N., \& Holman, M. J. 2008, ApJ, 677, 1324

Torres, G., Andersen, J., \& Giménez, A. 2010, A\&ARv, 18, 67

Zacharias, N., Monet, D. G., Levine, S. E., et al. 2005, VizieR Online Data Catalog, 1297, 0 